\title{
Bubble CPAP therapy for neonatal respiratory distress in level III neonatal unit in Amman, Jordan: a prospective observational study
}

This article was published in the following Dove Press journal: International Journal of General Medicine

\section{Manar Al-lawama' \\ Haitham Alkhatib' \\ Zaid Wakileh' \\ Randa Elqaisi' \\ Ghada AlMassad' \\ Eman Badran' \\ Tyler Hartman ${ }^{2}$}

'Pediatric Department, School of Medicine, Jordan University Hospital, The University of Jordan, Queen Rania Street, Amman I 1942, Jordan; ${ }^{2}$ Pediatric department, Geisel School of Medicine, Dartmouth University, Dartmouth-Hitchcock Medical Center, Lebanon, NH 03756, USA
Correspondence: Manar Al-lawama Pediatric Department, School of Medicine, Jordan University Hospital, The University of Jordan, Queen Rania Street, Amman II 942 , Jordan

Tel +962799068565

Emailm.allawama@ju.edu.jo
Purpose: Bubble continuous positive airway pressure (bCPAP), a noninvasive respiratory support modality used to manage newborns with respiratory distress, provides continuous pressure that helps prevent derecruitment of alveoli, increasing the lungs' functional residual capacity, and thus decreasing the work of breathing. bCPAP can be used to manage various respiratory conditions in the newborn. In this prospective study, we describe our experience using bCPAP therapy as the primary respiratory support in a level III neonatal unit in Amman, Jordan. In addition to reporting therapeutic indications, durations, and side effects, we aimed to identify areas requiring improvement in bCPAP therapy in our population.

Patients and methods: This prospective observational study investigated the usage of bCPAP in the management of respiratory distress in newborns admitted to a Jordan University Hospital in Amman. The newborns were followed until discharge. The patients' demographic and clinical data were recorded.

Results: A total of 143 babies (mean gestational age, 36 \pm 2.7 weeks; mean birth weight, $2,770 \pm 1,800 \mathrm{~g}$ ) were included. All received bCPAP as the primary respiratory support. The most common underlying cause of respiratory distress was transient tachypnea of the newborn (42\%), followed by prolonged respiratory transition (34\%). The therapy success rate was $93.7 \%$; only nine infants failed bCPAP. The most common side effect was physical facial injury.

Conclusion: The use of neonatal bCPAP therapy is well established in Jordan University Hospital. The area of potential improvement was the low rate of bCPAP use as a primary respiratory support in extremely premature infants.

Keywords: developing countries, transient tachypnea of the newborn, respiratory distress syndrome, postnatal adaptation

\section{Introduction}

Bubble continuous positive airway pressure (bCPAP), a noninvasive respiratory support modality used to manage newborns with respiratory distress, ${ }^{1}$ was first reported in 1971 to support the breathing of preterm neonates. ${ }^{2}$ bCPAP is less invasive than mechanical ventilation for delivering respiratory support to neonates. ${ }^{3}$

bCPAP provides continuous pressure that helps the collapse of alveoli on expansion, thereby increasing lung functional residual capacity and decreasing breathing work in neonates with respiratory distress. ${ }^{4}$ Moreover, bCPAP is relatively inexpensive and easy-to-use; accordingly, it is gaining popularity in developing countries as the method of choice for delivering CPAP to distressed neonates.$^{5-7}$ bCPAP can be used to manage various respiratory conditions in the newborn, including respiratory distress 
syndrome (RDS), transient tachypnea of the newborn (TTN), meconium aspiration, congenital pneumonia, pulmonary edema, and apnea. ${ }^{8}$ It can also be used in neonates postextubation to support the respiratory system and decrease the rate of reintubation. ${ }^{9}$

The Jordan University Hospital neonatal unit, a level III unit with a 30-bed capacity, provides care to sick newborns and premature infants. bCPAP was introduced to the unit in 2008. The service was expanded few years later by a neonatal service support project funded by The United States Agency for International Development. bCPAP therapy has since been primarily used to provide respiratory support to term and late preterm infants and for postextubation support in premature infants.

This is the first study from Jordan and the second from Arab countries to investigate the use of bCPAP in neonates. ${ }^{10}$ In this prospective study, our aim was to document the therapeutic use of bCPAP in neonatal patients in our part of the world and to describe our experience using bCPAP therapy as the primary respiratory support in a level III neonatal unit in Amman, Jordan. In addition to reporting therapeutic indications, durations, and side effects, we aim to identify areas of improvement in CPAP therapy in our population.

\section{Patients and methods}

This prospective observational study aimed to investigate the use of bCPAP in the management of respiratory distress in newborns admitted to the 30-bed neonatal unit of Jordan University Hospital in Amman, a tertiary center that provides $>4,000$ deliveries annually.

The present study, part of the "Using care bundles to improve neonatal health outcomes: JO-Neo study" project, was approved by the deanship of scientific research at the University of Jordan and the institutional review board and ethical committee of Jordan University Hospital (approval number 378/2017/10). It was conducted between January 2017 and December 2017. All procedures performed in this study were in accordance with the ethical standards of the Declaration of Helsinki 1964 and its later amendments. All the newborns included in the study received the standard of care and were followed prospectively; therefore, no consents were needed. The project was funded by Jordan University (grant number 2016-2017/48) and the scientific research support fund (grant number MPH/2/8/2016).

All neonates admitted to our unit with respiratory distress who received bCPAP therapy upon admission were included and followed until discharge. Neonates who were treated with CPAP as postextubation support were excluded. Demographic data and clinical data were recorded.

The available bCPAP device was manufactured by Fisher and Paykel, Auckland, New Zealand. The oxygen and air sources are piped oxygen and air that are then mixed to give the required fraction of inspired oxygen $\left(\mathrm{FiO}_{2}\right)$. The mixed oxygen is humidified and delivered at a flow rate of $6 \mathrm{~L} / \mathrm{min}$ and pressure of $6 \mathrm{~cm} \mathrm{H}_{2} \mathrm{O}$. During the study period, the interfaces used varied between (Fisher and Paykel) nasal cannula and masks, and (Great Group Medical Co., LTD., Changhua County, Taiwan) infant nasal prongs.

Respiratory distress is defined as the presence of one or more of the following signs: tachypnea with a respiratory rate $>60$ breaths/min, grunting, retractions, flaring ala nasi, cyanosis, and/or apnea. ${ }^{11}$ TTN is defined as the presence of mild respiratory distress that occurs primarily in term and late preterm infants within 2 hours of birth that resolves within 72 hours. ${ }^{12}$ Prolonged respiratory transition $(\mathrm{PT})^{13}$ is defined here as a separate respiratory entity from TTN as the presence of respiratory distress signs at birth that usually improves with the use of CPAP therapy within $<6$ hours of birth. RDS is the presence of respiratory distress signs within the first 6 hours of life, especially in preterm infants with characteristic radiological findings that usually persist for more than 2-3 days. ${ }^{14}$ Noninvasive positive pressure ventilation (NIPPV) is defined as ventilating the newborn by conventional mechanical ventilation using continuous mandatory ventilation mode with the same setting used in mechanical ventilation but using nasal prongs as the interface. ${ }^{15}$ Failure of CPAP therapy is defined as the need for endotracheal intubation or escalation of therapy to NIPPV due to the persistence of severe respiratory distress signs and the need for a high oxygen concentration ( $>40 \%$ ) despite CPAP therapy with a pressure of $6 \mathrm{~cm} \mathrm{H}_{2} \mathrm{O}$. bCPAPs were not routinely increased before switching to NIPPV therapy.

CPAP therapy is ordered by the physician but applied and monitored by neonatal unit nurses. There is no unit protocol for the nursing care of newborns on CPAP. Machine maintenance is provided by respiratory therapists. Weaning from CPAP therapy can be initiated by the bedside nurse or by physicians. Weaning depends on the resolution of the respiratory distress signs and when $\mathrm{FiO}_{2}$ reaches $21 \%-30 \%$. $\mathrm{FiO}_{2}$ is tapered down by the bedside nurse according to oxygen saturation. Newborns are usually weaned off at a pressure of $6 \mathrm{~cm} \mathrm{H}_{2} \mathrm{O}$. If weaning fails, the pressure is gradually lowered to $4-5 \mathrm{~cm} \mathrm{H}_{2} \mathrm{O}$.

In this study, in the delivery room, CPAP was provided using a T-piece resuscitator with a pressure of $5 \mathrm{~cm} \mathrm{H}_{2} \mathrm{O}$ until transfer to the neonatal unit using the same device connected 
to a pressurized $100 \%$ oxygen cylinder. In the neonatal unit, nasal bCPAP was applied by the bedside nurse and the newborn was placed in a neutral thermal environment and connected to a continuous cardiorespiratory monitor. Vital signs and respiratory distress signs were assessed and documented hourly. The newborn was screened for risk factors for infection or other perinatal morbidities through a detailed history and physical examination. If respiratory distress symptoms were resolved within 6 hours, feeding was started and the infant was transferred back to the mother's room.

A chest radiograph was performed in cases in which the respiratory symptoms persisted for $>6$ hours unless a pneumothorax or congenital anomaly is suspected. Surfactant therapy was provided according to the INSURE (intubate, surfactant, extubate) protocol as a rescue therapy after CPAP failure and a trial of NIPPV.

Empirical antibiotic treatment was reserved for newborns with respiratory distress who were clinically sick, if the infant with respiratory distress has delayed capillary refill, temperature instability, or recurrent apnea, and he or she would be considered sick. For newborns with respiratory distress who were otherwise well and whose symptoms persisted for $>6$ hours of age, a complete blood count and $\mathrm{C}$-reactive protein level were determined at 6-12 hours of age. Empirical antibiotics were administered if abnormal laboratory results were obtained.

\section{Statistical analysis}

Mean and standard deviations are provided for continuous variables, while frequencies and relative percentages are reported for categorical variables.

\section{Results}

A total of 143 babies who received bCPAP as the primary respiratory support were included in the study and analyses. Their mean gestational age was $36 \pm 2.7$ weeks. Mean birth weight was $2,770 \pm 1,800 \mathrm{~g}$. Most of the included newborns were male (64\%). The patients' detailed demographic characteristics are shown in Table 1.

Grunting was the most common presenting sign of respiratory distress $(83 \%)$, followed by tachypnea ( $59 \%)$. The most common underlying cause of respiratory distress was TTN (42\%), followed by PT (34\%) (Table 2).

Respiratory distress started at birth in most of the infants (97\%). Most of the included infants required CPAP therapy for 6-48 hours. Feeding while on CPAP (either suckling or by orogastric tube) was initiated in $48 \%$ of newborns who needed CPAP therapy for $>6$ hours. Nurses initiated the CPAP weaning in $50 \%$ of cases (Table 3 ).
Table I Demographic characteristics of neonates with respiratory distress treated with nCPAP

\begin{tabular}{|l|l|}
\hline Characteristics & No (\%) \\
\hline Gestational age (mean \pm SD) & $36 \pm 2.7$ \\
\hline Term & $78(54)$ \\
\hline Late preterm & $48(34)$ \\
\hline Preterm & $17(12)$ \\
\hline Birth weight (mean \pm SD) & $2.77 \pm 1.8$ \\
\hline Male gender & $92(64)$ \\
\hline Twin gestation & $18(12.5)$ \\
\hline Cesarean section & $99(69)$ \\
\hline First minute & 7 \\
\hline Fifth minute & 9 \\
\hline Resuscitation details & \\
\hline Positive pressure ventilation & $20(14)$ \\
\hline Chest compression & $1(0.7)$ \\
\hline Maternal age (mean) & 30.4 \\
\hline Maternal diabetes & $17(12)$ \\
\hline Maternal hypertension & $9(6)$ \\
\hline Infection risk factors & $7(5)$ \\
\hline Prolonged rupture of membranes & \\
\hline Group B Strep status & $2(\mathrm{I})$ \\
\hline Positive & $14 \mathrm{I}(99)$ \\
\hline Unknown & $52(36)$ \\
\hline Maternal urinary tract infection & \\
\hline
\end{tabular}

Abbreviations: nCPAP, nasal continuous positive airway pressure; Strep, streptococcus.

Table 2 Respiratory distress signs and the underlying lung pathology of neonates treated with nCPAP

\begin{tabular}{|l|l|}
\hline & No (\%) \\
\hline Respiratory distress signs & \\
\hline Tachypnea & $85(59)$ \\
\hline Grunting & $120(83)$ \\
\hline Apnea & $3(2)$ \\
\hline Cyanosis & $18(12)$ \\
\hline Retraction & $30(20)$ \\
\hline Underlying lung pathology & $49(34)$ \\
\hline Prolonged transition & $61(42)$ \\
\hline Transient tachypnea & $33(23)$ \\
\hline Respiratory distress syndrome &
\end{tabular}

Abbreviation: nCPAP, nasal continuous positive airway pressure.

Of the newborns with a PT, $63 \%$ were born via cesarean section. Their mean gestational age was $37 \pm 1.5$ weeks, mean birth weight was $2,900 \pm 390 \mathrm{~g}$, and average duration of CPAP therapy was $4.5 \pm 1.5$ hours (Table 4 ).

In newborns with TTN, $69 \%$ were born via cesarean section. Their mean gestational age was $36 \pm 1.7$ weeks, mean birth weight was $3,000 \pm 390 \mathrm{~g}$, and average duration of CPAP therapy was $21 \pm 10$ hours (Table 4 ).

Of the newborns with RDS, $84 \%$ were born via cesarean section. Their mean gestational age was $34 \pm 2.5$ weeks, mean 
birth weight was $2,600 \pm 1,100 \mathrm{~g}$, and average duration of CPAP therapy was $18 \pm 13$ days (Table 4 ).

The most commonly encountered CPAP complication was facial physical injuries, eye puffiness being the most common (11\%) (Table 5).

\section{Discussion}

This prospective observational study included all newborns with respiratory distress who were treated with nasal bCPAP as the primary respiratory support. This is the first study from Jordan to report on the use of nasal bCPAP.

Table 3 Characteristics of nCPAP therapy in neonates with respiratory distress

\begin{tabular}{|c|c|}
\hline Characteristics & No (\%) \\
\hline \multicolumn{2}{|l|}{ Starting age of CPAP therapy } \\
\hline At birth & $140(97)$ \\
\hline$>6$ hours of age & $5(3)$ \\
\hline \multicolumn{2}{|l|}{$\mathrm{FiO}_{2}$ needs upon CPAP start } \\
\hline$<40 \%$ & $135(93)$ \\
\hline$>40 \%$ & $10(7)$ \\
\hline \multicolumn{2}{|l|}{ Interface type } \\
\hline Nasal mask & $80(55)$ \\
\hline Traditional nasal prongs & $61(42)$ \\
\hline Horizontal nasal prongs & $3(2)$ \\
\hline Orogastric tube in situ & $143(100)$ \\
\hline Feeding while on CPAP & $44 / 94(47)$ \\
\hline \multicolumn{2}{|l|}{ CPAP therapy duration } \\
\hline$<6$ hours & $43(30)$ \\
\hline 6 hours to 2 days & $64(44)$ \\
\hline $2-14$ days & $36(25)$ \\
\hline 14 days to 2 months & $2(I)$ \\
\hline \multicolumn{2}{|c|}{ CPAP therapy duration according to diagnosis } \\
\hline Prolonged transition & $4.5 \pm 1.5$ hours \\
\hline Transient tachypnea of newborns & $21 \pm 10$ hours \\
\hline Respiratory distress syndrome & $18 \pm 13$ days \\
\hline Nurse initiated weaning & $72(50)$ \\
\hline Physician initiated weaning & $71(50)$ \\
\hline Failed CPAP & $9(6)$ \\
\hline Surfactant therapy & $\mathrm{I}(0.6)$ \\
\hline Length of hospital stay & $7 \pm 5.7$ \\
\hline
\end{tabular}

Abbreviation: $\mathrm{nCPAP}$, nasal continuous positive airway pressure.
Our unit has been providing bCPAP therapy for the past 9 years as the primary mode of ventilation support, mostly in term and late preterm infants and as postextubation support for extremely premature infants. This study was conducted to evaluate our experience with bCPAP use as the primary ventilation support and identify areas requiring improvement.

The use of CPAP therapy in developing countries is well documented. ${ }^{16}$ The fact that it is less expensive, ${ }^{3}$ has low maintenance needs, and its use does not require a high skill level ${ }^{4}$ makes it an excellent option in areas with low resources like Jordan.

In our study, the included newborns were mostly term (54\%) and late preterm (33\%) infants. Only $12 \%$ of our cohort consisted of preterm infants with a gestational age of 29-33 weeks. The included newborns were relatively well and did not need resuscitation at birth. The success rate of CPAP therapy was high (93.7\%), with only nine newborns failing CPAP and requiring respiratory support using NIPPV; five of these infants had a gestational age $<34$ weeks. Only one newborn required intubation and surfactant therapy.

CPAP was not used as the primary mode of ventilation in babies $<28$ weeks' gestation. A previous study found that only one-third of very low birth weight infants in our unit were primarily supported using CPAP. ${ }^{17}$ This is an area that requires effort to build the culture and confidence of not intubating extremely premature infants.

Respiratory distress causes in the included newborns were variable and similar to previously reported numbers from different areas in the world, TTN was the most common

Table 5 Complications of nCPAP therapy in neonates in a tertiary care center in Amman, Jordan

\begin{tabular}{|l|l|}
\hline Complications & No (\%) \\
\hline Nasal septum injury & $2(\mathrm{I})$ \\
\hline Nasal bridge injury & $4(3)$ \\
\hline Eye puffiness and hyperemia & $16(\mathrm{II})$ \\
\hline Abdominal distention & $2(\mathrm{I})$ \\
\hline Pneumothorax & $0(0)$ \\
\hline
\end{tabular}

Abbreviation: $\mathrm{nCPAP}$, nasal continuous positive airway pressure.

Table 4 Comparison between neonatal clinical characteristics according to diagnosis

\begin{tabular}{|l|l|l|l|}
\hline Clinical characteristics & $\begin{array}{l}\text { Prolonged } \\
\text { transition }\end{array}$ & $\begin{array}{l}\text { Transient tachypnea } \\
\text { of newborns }\end{array}$ & $\begin{array}{l}\text { Respiratory distress } \\
\text { syndrome }\end{array}$ \\
\hline Gestational age (mean \pm SD), week & $37 \pm I .5$ & $36 \pm I .7$ & $34 \pm 2.5$ \\
\hline Birth weight (mean \pm SD), g & $2,900 \pm 390$ & $3,000 \pm 390$ & $2,600 \pm I, 100$ \\
\hline Male gender & $36 / 44(8 I)$ & $34 / 6 I(56)$ & $22 / 33(67)$ \\
\hline Cesarean section & $3 I / 44(70)$ & $4 I / 6 I(67)$ & $27 / 33(8 I)$ \\
\hline nCPAP duration & $4.5 \pm I .5$ hours & $2 I \pm 10$ hours & $18 \pm 13$ days \\
\hline
\end{tabular}

Abbreviation: nCPAP, nasal continuous positive airway pressure. 
cause (42\%), followed by PT in 34\%, and then the third most common cause was RDS in $23 \%$. The clinical characteristics of the included infants and the duration of CPAP therapy were variable based on the original lung disease (Table 4).

PT is one of the main features of postnatal maladaptation. ${ }^{13}$ The main issue in these infants is the delay in the absorption of fetal lung fluid, ${ }^{18}$ which is usually triggered by the rapid endocrine changes occurring perinatally. This process might be delayed in infants born via cesarean section especially if the mother did not go into labor. ${ }^{19}$ This explains our observations of both TTN and PT in this study. Providing continuous distending pressure via nasal bCPAP therapy will stabilize the alveoli, prevent their collapse, and help stabilize surfactant at the alveolar surfaces; as a result, it improves functional residual capacity and tidal volumes. ${ }^{20}$ Thirty-four percent of our cohort fell into this category; most (63\%) were delivered via cesarean section. They were relatively mature newborns with a mean gestational age of $37 \pm 1.5$ vs $36 \pm 1.7$ and $34 \pm 2.5$ weeks for those with TTN and RDS, respectively. The average duration of CPAP therapy for newborns with PT was $4.5 \pm 1.5$ hours. For all these infants, $\mathrm{FiO}_{2}$ requirements were below $40 \%$. None of these infants had complications. In our study, providing bCPAP via a nasal cannula was vital to a smooth respiratory transition.

TTN is a relatively benign respiratory morbidity characterized by mild respiratory distress symptoms that usually resolve within a few days. ${ }^{21}$ It is the most common respiratory morbidity in term and late preterm infants, ${ }^{22}$ especially after elective cesarean section. ${ }^{23}$ The underlying pathophysiology of TTN is a delayed absorption of the lung fluid. ${ }^{12}$ The use of CPAP therapy in newborns with TTN is well documented. ${ }^{24}$

In our cohort, TTN was the most common respiratory morbidity $(42 \%)$. The mean gestational age of newborns with TTN was $36 \pm 1.7$ weeks. Most (69\%) were delivered by cesarean section. For all these infants, $\mathrm{FiO}_{2}$ requirements were below $40 \%$. The average length of CPAP therapy was $21 \pm 10$ hours.

Respiratory distress of the newborn is primarily a disease of prematurity, as its main pathophysiological issue is surfactant deficiency. ${ }^{25}$ As a result, lung compliance is decreased and the newborn needs to generate more pressure to achieve the required tidal volumes. ${ }^{26}$ Without the appropriate respiratory support, affected newborns will develop hypoxemia and acidosis, which will initiate an inflammatory reaction that will further harm the lung epithelium. ${ }^{27} \mathrm{CPAP}$ helps splint the airways of premature infants and decrease the rate of apnea. ${ }^{28}$
RDS, the third most common respiratory morbidity noted in our cohort (33\%), affected more premature infants with a mean gestational age of $34 \pm 2.5$ weeks. Most ( $81 \%)$ were delivered via cesarean section. Their average length of bCPAP therapy was much longer than those in the PT and TTN groups (18 \pm 13 days) as expected from their maturity level. ${ }^{29}$ The bCPAP weaning criteria for premature infants are ill defined, and postmenstrual age, weight, and methods are not agreed upon in our unit. Defining bCPAP weaning criteria for premature infants is another area requiring improvement.

Studies from other developing countries included fewer numbers of patients, ${ }^{8,10,16}$ but similar gestational age categories as our patients, the distribution of respiratory distress causes was similar. However, these studies compared nCPAP as a new modality of treatment with their standard of care, while in our institution nCPAP is the standard of care treatment for all neonates with respiratory distress.

Numerous challenges arise when caring for premature infants who are expected to need prolonged CPAP therapy. Feeding, positioning, developmental care, and kangaroo care while on CPAP are the main challenges. ${ }^{30}$ Avoiding nasal injury is also another challenge. ${ }^{31}$ Much experience is required to overcome these challenges.

Among the newborns who needed CPAP therapy for $>6$ hours, feeding was established in $48 \%$. Most of our included infants were born via cesarean section, and waiting for the mothers to be mobilized to initiate breastfeeding explained the feeding delays in most cases. CPAP weaning was initiated by nurses in $50 \%$ of cases, which is a reflection of the level of experience our nursing staff has developed over the past 9 years. The most commonly seen side effect of CPAP in our setting was physical facial injuries.

\section{Conclusion}

This study shed light on neonatal respiratory support care provided in our part of the world. It shows that neonatal bCPAP therapy is well established in Jordan University Hospital, with a success rate of $93.7 \%$. Identified areas of potential improvement included the low rate of CPAP use in extreme premature infants as a primary respiratory support, the absence of a monitoring protocol for newborns while on CPAP, and the lack of weaning criteria for premature infants on CPAP. These factors should be included in a CPAP improvement project. To this end, Jordan University Hospital can function as a training center for bCPAP therapy for centers nationwide. 


\section{Data sharing statement}

The data sets used and/or analyzed during the current study are available from the corresponding author on reasonable request.

\section{Acknowledgments}

We thank Miss Wafa Rabayaa for her efforts and dedication in following these infants and gathering their data. We thank all of our nursing staff, who have major contribution to our CPAP respiratory care success. The project was funded by Jordan University grant number 2016-2017/48 and by the scientific research support fund grant number MPH/2/8/2016.

\section{Disclosure}

The authors report no conflicts of interest in this work.

\section{References}

1. Millar D, Kirpalani H. Benefits of non invasive ventilation. Indian Pediatr. 2004;41(10):1008-1011.

2. Gregory GA, Kitterman JA, Phibbs RH, Tooley WH, Hamilton WK. Treatment of the idiopathic respiratory-distress syndrome with continuous positive airway pressure. N Engl J Med. 1971;284(24):1333-1340.

3. Nowadzky T, Pantoja A, Britton JR. Bubble continuous positive airway pressure, a potentially better practice, reduces the use of mechanical ventilation among very low birth weight infants with respiratory distress syndrome. Pediatrics. 2009;123(6):1534-1540.

4. Upadhyay A, Deorari AK. Continuous positive airway pressure - a gentler approach to ventilation. Indian Pediatr. 2004;41(5):459-469.

5. Rezzonico R, Caccamo LM, Manfredini V, et al. Impact of the systematic introduction of low-cost bubble nasal CPAP in a NICU of a developing country: a prospective pre- and post-intervention study. BMC Pediatr. 2015;15:26.

6. Dewez JE, van den Broek N. Continuous positive airway pressure (CPAP) to treat respiratory distress in newborns in low- and middleincome countries. Trop Doct. 2017;47(1):19-22.

7. Jayashree M, KiranBabu HB, Singhi S, Nallasamy K. Use of nasal bubble CPAP in children with hypoxemic clinical pneumonia-report from a resource limited set-up. J Trop Pediatr. 2016;62(1):69-74.

8. Abdulkadir I, Hassan L, Abdullahi F, Purdue S, Ogala WN. Nasal bubble CPAP: one year experience in a neonatal unit of a tertiary health facility in northwestern Nigeria. Niger Postgrad Med J. 2015;22(1):21-24.

9. Cummings JJ, Polin RA; The Committee on Fetus and Newborn, American Academy of Pediatrics. Noninvasive respiratory support. Pediatrics. 2016;137(1):169-73.

10. Bassiouny MR, Gupta A, El Bualy M. Nasal continuous positive airway pressure in the treatment of respiratory distress syndrome: an experience from a developing country. J Trop Pediatr. 1994;40(6):341-344.

11. Gallacher DJ, Hart K, Kotecha S. Common respiratory conditions of the newborn. Breathe (Shef). 2016;12(1):30-42.
12. Dehdashtian M, Aletayeb M, Malakian A, Aramesh MR, Malvandi H. Clinical course in infants diagnosed with transient tachypnea of newborn: a clinical trial assessing the role of conservative versus conventional management. J Chin Med Assoc. 2018;81(2):183-186.

13. Sinha SK, Donn SM. Fetal-to-neonatal maladaptation. Semin Fetal Neonatal Med. 2006;11(3):166-173.

14. Reuter S, Moser C, Baack M. Respiratory distress in the newborn. Pediatr Rev. 2014;35(10):417-428; quiz 429.

15. Roberts CT, Davis PG, Owen LS. Neonatal non-invasive respiratory support: synchronised NIPPV, non-synchronised NIPPV or bi-level CPAP: what is the evidence in 2013? Neonatology. 2013;104(3):203-209.

16. Kawaza K, Machen HE, Brown J, et al. Efficacy of a low-cost bubble CPAP system in treatment of respiratory distress in a neonatal ward in Malawi. PLoS One. 2014;9(1):e86327.

17. Al-lawama M, Abdelghani T, Badran E. Mortality and short-term outcomes of very low birth weight infants at a tertiary care center in Jordan: comparison with other countries. Pediatr Ther. 2017;7:311.

18. Askin DF. Fetal-to-neonatal transition - what is normal and what is not? Neonatal Netw. 2009;28(3):e33-e40.

19. Hillman NH, Kallapur SG, Jobe AH. Physiology of transition from intrauterine to extrauterine life. Clin Perinatol. 2012;39(4):769-783.

20. Gupta S, Donn SM. Continuous positive airway pressure: to bubble or not to bubble? Clin Perinatol. 2016;43(4):647-659.

21. Liu J, Wang Y, Fu W, Yang CS, Huang JJ. Diagnosis of neonatal transient tachypnea and its differentiation from respiratory distress syndrome using lung ultrasound. Medicine (Baltimore). 2014;93(27):e197.

22. Osman AM, El-Farrash RA, Mohammed EH. Early rescue neopuff for infants with transient tachypnea of newborn: a randomized controlled trial. J Matern Fetal Neonatal Med. 2017;16:1-7.

23. Celebi MY, Alan S, Kahvecioglu D, et al. Impact of prophylactic continuous positive airway pressure on transient tachypnea of the newborn and neonatal intensive care admission in newborns delivered by elective cesarean section. Am J Perinatol. 2016;33(1):99-106.

24. Yagui AC, Vale LA, Haddad LB, et al. Bubble CPAP versus CPAP with variable flow in newborns with respiratory distress: a randomized controlled trial. J Pediatr (Rio J). 2011;87(6):499-504.

25. Rubarth LB, Quinn J. Respiratory development and respiratory distress syndrome. Neonatal Netw. 2015;34(4):231-238.

26. Mahoney AD, Jain L. Respiratory disorders in moderately preterm, late preterm, and early term infants. Clin Perinatol. 2013;40(4):665-678.

27. Jones M. Effect of preterm birth on airway function and lung growth. Paediatr Respir Rev. 2009;10 (Suppl 1):9-11.

28. Miller MJ, DiFiore JM, Strohl KP, Martin RJ. Effects of nasal CPAP on supraglottic and total pulmonary resistance in preterm infants. $J$ Appl Physiol (1985). 1990;68(1):141-146.

29. Bamat N, Jensen EA, Kirpalani H. Duration of continuous positive airway pressure in premature infants. Semin Fetal Neonatal Med. 2016;21(3):189-195.

30. Hanin M, Nuthakki S, Malkar MB, Jadcherla SR. Safety and efficacy of oral feeding in infants with BPD on nasal CPAP. Dysphagia. 2015;30(2):121-127.

31. Imbulana DI, Manley BJ, Dawson JA, Davis PG, Owen LS. Nasal injury in preterm infants receiving non-invasive respiratory support: a systematic review. Arch Dis Child Fetal Neonatal Ed. 2018;103(1): F29-F35.
International Journal of General Medicine

\section{Publish your work in this journal}

The International Journal of General Medicine is an international, peer-reviewed open-access journal that focuses on general and internal medicine, pathogenesis, epidemiology, diagnosis, monitoring and treatment protocols. The journal is characterized by the rapid reporting of reviews, original research and clinical studies across all disease areas.

\section{Dovepress}

The manuscript management system is completely online and includes a very quick and fair peer-review system, which is all easy to use. Visit http://www.dovepress.com/testimonials.php to read real quotes from published authors. 